\title{
NONLINEAR DYNAMICS AND WALL TOUCH-UP IN UNSTABLY STRATIFIED MULTILAYER FLOWS IN HORIZONTAL CHANNELS UNDER THE ACTION OF ELECTRIC FIELDS*
}

\author{
LYUDMYLA L. BARANNYK ${ }^{\dagger}$, DEMETRIOS T. PAPAGEORGIOU ${ }^{\ddagger}$, PETER G. \\ PETROPOULOS ${ }^{\S}$, AND JEAN-MARC VANDEN-BROECK $\$$
}

\begin{abstract}
This study considers the nonlinear dynamics of stratified immiscible fluids when an electric field acts perpendicular to the direction of gravity. A particular setup is investigated in detail, namely, two stratified fluids inside a horizontal channel of infinite extent. The fluids are taken to be perfect dielectrics, and a constant horizontal field is imposed along the channel. The sharp interface separating the two fluids may or may not support surface tension, and the RayleighTaylor instability is typically present when the heavier fluid is on top. A novel system of partial differential equations that describe the interfacial position and the leading order horizontal velocity in the fluid layers is studied analytically and computationally. The system is valid in the asymptotic limit of one layer being asymptotically thin compared to the second fluid layer, and as a result nonlocal electrostatic terms arise due to the multiscale nature of the physical setup. The initial value problem on spatially periodic domains is solved numerically, and it is shown that a sufficiently strong electric field can linearly stabilize the Rayleigh-Taylor instability to produce nonlinear quasiperiodic oscillations in time that are quite close to standing waves. In situations when the instability is present, the system is shown to generically evolve to touch-up singularities with the interface touching the upper wall in finite time while the leading order horizontal velocity blows up. Accurate numerical solutions allied with asymptotic analysis show that the terminal states follow self-similar structures that are different if surface tension is present or absent, but with the electric field present. In the presence of surface tension, the touch-up is found to take place with bounded interfacial gradients but unbounded curvature, with electrostatic effects relegated to higher order. If surface tension is absent, however, the electric field supports touch-up with a local cusp structure so that the interfacial gradients themselves are unbounded. The self-similar solutions are of the second kind and extensive simulations are used to extract the scaling exponents. Distinct and independent methods are described and implemented, and agreement between them is excellent.
\end{abstract}

Key words. singularity formation, similarity solutions, finite time singularity, touch-up singularity, Rayleigh-Taylor instability, electric fields, asymptotic behavior, Fourier analysis

AMS subject classifications. 76M55, 76M45, 76M22, 76E25, 76E17

DOI. $10.1137 / 140968070$

1. Introduction. Rayleigh-Taylor instability is a fundamental phenomenon in fluid dynamics, and it occurs when a heavier fluid lies above a lighter fluid in the presence of a constant gravitational field [30, 35]. Interest in this problem stems from its importance to a wide variety of applications such as oceanography, mixing

${ }^{*}$ Received by the editors May 6, 2014; accepted for publication (in revised form) October 27, 2014; published electronically January 15, 2015.

http://www.siam.org/journals/siap/75-1/96807.html

†Department of Mathematics, University of Idaho, Moscow, ID 83844 (barannyk@uidaho.edu). This author's work was supported by the National Science Foundation Major Research Instrumentation Program, grant 1229766, as well as by Idaho National Laboratory.

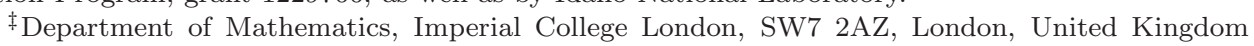
(d.papageorgiou@imperial.ac.uk). This author's work partially supported by the National Science Foundation grant DMS-0707339 and by the Engineering and Physical Sciences Research Council of Great Britain under grant EP/K041134/1.

$\S$ Department of Mathematical Sciences, New Jersey Institute of Technology, Newark, NJ (peterp@ njit.edu). This author's work was partially supported by the National Science Foundation grant DMS-0707339.

IDepartment of Mathematics, University College London, London WC1E 6BT, UK (j.vandenbroeck@ucl.ac.uk).

92

Copyright @ $\odot$ by SIAM. Unauthorized reproduction of this article is prohibited. 
([2] and references therein), thermal convection [10], finger selection in splashes [17], inertial confinement fusion [39,31,3], and other natural phenomena and technical applications [32]. When fluids are immiscible, a sharp interface exists between them and any perturbation of the interface causes formation of a pattern of rising bubbles of lighter fluid and falling spikes of heavier fluid. As the lighter and heavier fluids pass by each other, a strong shearing flow develops on the sides of the spike. This part of the interface is then susceptible to the Kelvin-Helmholtz instability. For inviscid and incompressible fluids, the shear region and the interface coincide and the interface can be modeled as a vortex sheet with the strength changing by the baroclinic generation of vorticity [4]. The growth rate in the Kelvin-Helmholtz instability is higher than than in Rayleigh-Taylor instability. The Kelvin-Helmholtz instability dominates and, as a result, the vortex sheet rolls up. The type of subsequent roll-up may be related to the type of singularity developed by the interface. It is believed that these singularities immediately precede the rollup of the interface and determine the rate of mixing in the flow $[4,33]$. A recent review of theoretical modeling approaches of Rayleigh-Taylor instability and turbulent mixing is provided by [1].

Singularity formation in interfacial flows has received considerable attention due to the fact that singularities, if encountered, are important mathematically and physically. The singularities considered here are different from those found in vortex sheet or Rayleigh-Taylor problems (see, for example, [24, 4, 23, 8, 37]). Instead of a curvature singularity, the confining geometry in the present study induces fluid-structure interaction and touch-up singularities in finite time. The behavior is more reminiscent of the dynamics of liquid layer flows as in $[27,36]$ for inviscid electrified flows, and $[38,40]$ for viscous electrified flows. The physical origin of the instabilities is different, however, but the mathematical singularities after finite time are analogous to those described here. In the liquid layer problem in [27], gravity is stabilizing, but a vertical electric field induces instability that manifests itself nonlinearly by a touchdown in finite time with corner singularities in the presence of surface tension. The liquid sheet study in [36] has an electric field parallel to the undisturbed sheet surface, and gravitational forces are absent; the flow is linearly stable but can be pushed to rupture by a sufficiently large initial condition - surface tension is present and corner singularities emerge once more. Both of these cases are analogous to the present study when surface tension is present. The problem where surface tension is absent but an electric field is used to stabilize the system has not been studied previously and in fact is shown to produce stronger spatial singularities in the sense that the interface touches the wall with a cusp form locally. From a physical perspective, it is useful to identify the type of emerging short-scale structures because they affect the rate at which the electric field becomes singular. Large electric fields can in turn produce dielectric breakdown, a phenomenon that is quite complex to model accurately.

Linearly, Rayleigh-Taylor instability in ideal fluids has short waves growing the fastest (see Chandrasekhar [11]). These can be dispersively regularized by the inclusion of surface tension, and the flow can be followed into the nonlinear regime (see, for example, the models, analysis, and computations in $[29,5,7,12])$. However, in general situations surface tension is not sufficient to completely suppress the Rayleigh-Taylor instability, which is still dominant in the long wave regime. In this paper, we investigate the effects of horizontally applied electric fields that have been shown to also induce a dispersive regularization but in particular can completely suppress RayleighTaylor instability in the linear regime at least $[14,22,13,20]$. Nonlinear studies were considered in [6], and direct numerical simulations of the Navier-Stokes equations coupled to electrostatics have been carried out in [13]. The latter paper also demonstrates 
how the electric field can be used (by on-off switching) to generate sustained periodic interfacial oscillations that can be useful in mixing. The nonlinear study [6] excludes Kelvin-Helmholtz instability and shows that a horizontal electric field can completely suppress Rayleigh-Taylor instability in bounded two-fluid systems. The related study [20] considers a hanging film wetting the underside of a horizontal dielectric slab; beneath the film there is an unbounded hydrodynamically passive dielectric (e.g., air) and the effect of the solid dielectric slab is taken into account, i.e., the electric field is solved for in that region also to provide a nonlocal effective boundary condition for the field at the horizontal fluid-solid interface. It is shown that the electric field can suppress the gravitational instability in agreement with the present model. However, if surface tension forces and/or electric fields are weak, the interface is expected to develop a singularity after a finite time, and the detailed mathematical structures of such solutions are the subject of the present work.

The paper is organized as follows. In section 2, we briefly formulate the problem and derivation of evolution equations for thin upper layers. Linear stability results are presented in section 3 to demonstrate that both surface tension and/or the electric field can completely suppress Rayleigh-Taylor instability. Representative numerical solutions in stable and unstable regimes are shown in section 4 . They indicate that when surface tension and/or the electric field are not strong enough, the instability develops after a finite time with the interface touching the wall and velocity blowing up. Section 5 is devoted to the construction of similarity solutions that describe such terminal singular states. The singularity is shown to be of the second type and the exponents depend on a parameter $\alpha$. Three different methods to estimate the value of $\alpha$ are presented. They are based either on temporal or spatial structure of singularity and produce accurate approximations of $\alpha$ with an excellent agreement. The cases when surface tension is present or absent are considered separately since scalings are different. Section 6 provides our conclusions.

\section{Mathematical model.}

2.1. Governing equations and boundary conditions. We consider a twodimensional system of two immiscible, inviscid, irrotational, and incompressible fluids with densities $\rho_{1}, \rho_{2}$ that are bound together in an infinite horizontal channel of depth $D_{u}$ and separated by an interface given by $y=H(x, t)$ in a Cartesian coordinate system with $t$ being time. The schematic of the problem is shown in Figure 1. The fluids are assumed to be perfect dielectrics that have electrical permittivities $\epsilon_{1}$ and $\epsilon_{2}$, and the surface tension coefficient between them is $\sigma$. The lower fluid is located in region 1 given by $0<y<H(x, t)$ and the upper fluid occupies region 2 given by $H(x, t)<y<D_{u}$. A uniform electric field $\mathbf{E}_{0}=E_{0} \hat{\mathbf{i}}=\frac{V_{0}}{D} \hat{\mathbf{i}}$ acts horizontally with respect to the undisturbed configuration, with $V_{0}$ the characteristic voltage drop and $D$ the undisturbed depth of the lower layer. Gravity acts in the negative $y$ direction with acceleration $g$. The hydrodynamic equations are derived from Euler's equations, which can be represented in terms of harmonic fluid potentials $\phi_{1}$ and $\phi_{2}$, say, with the fluid velocity fields given by $\mathbf{u}_{1,2}=\nabla \phi_{1,2}$. The electrodynamic equations are obtained from the electrostatic limit of Maxwell's equations. Since the induced magnetic fields are negligible, Faraday's law becomes $\nabla \times \mathbf{E}_{1,2}=0$, where $\mathbf{E}_{1,2}$ is the electric field in each region. Therefore, we can introduce voltage potentials $V_{1,2}$ such that $\mathbf{E}_{1,2}=-\nabla V_{1,2}$. In addition, because there are no volumetric charge concentrations in the fluids, Gauss's law reads $\nabla \cdot\left(\epsilon_{1,2} \mathbf{E}_{1,2}\right)=0$. Combining this with Faraday's law, we get that $V_{1}$ and $V_{2}$ are also harmonic functions. The field equations

Copyright @ $\odot$ by SIAM. Unauthorized reproduction of this article is prohibited. 


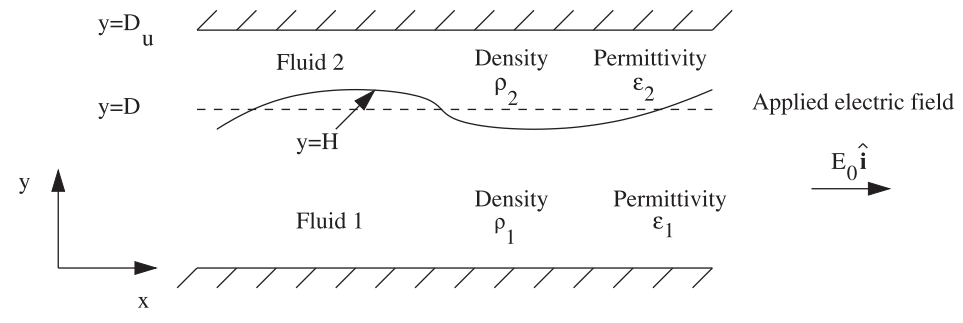

FIG. 1. Schematic of the problem.

are, then,

$$
\Delta \phi_{1,2}=0, \quad \Delta V_{1,2}=0,
$$

where $\Delta \equiv \frac{\partial}{\partial x^{2}}+\frac{\partial}{\partial y^{2}}$. The boundary conditions at $y=H(x, t)$ are the kinematic conditions, continuity of normal stresses, continuity of the normal component of the displacement field $\epsilon \mathbf{E}$, and continuity of the tangential component of the electric field:

$$
\begin{gathered}
H_{t}+\phi_{1 x} H_{x}-\phi_{1 y}=0, \quad H_{t}+\phi_{2 x} H_{x}-\phi_{2 y}=0, \\
{[\mathbf{n} \cdot \mathbf{T} \cdot \mathbf{n}]_{2}^{1}=\sigma \nabla_{s} \cdot \mathbf{n}} \\
{[\epsilon \mathbf{E} \cdot \mathbf{n}]_{2}^{1}=0, \quad \mathbf{n} \times[\mathbf{E}]_{2}^{1}=0}
\end{gathered}
$$

where $[\cdot]_{2}^{1}$ denotes the jump in the quantity as the interface is crossed from the lower to the upper fluid, and $\mathbf{n}=\frac{\left(-H_{x}, 1\right)}{\left(1+H_{x}^{2}\right)^{1 / 2}}, \mathbf{t}=\frac{\left(1, H_{x}\right)}{\left(1+H_{x}^{2}\right)^{1 / 2}}$ are the unit normal (pointing out of region 1) and tangent to the interface, respectively. The stress tensor $\mathbf{T}$ is defined by

$$
T_{i j}=-p \delta_{i j}+\epsilon\left(E_{i} E_{j}-\frac{1}{2}|\mathbf{E}|^{2} \delta_{i j}\right)
$$

where the first term represents the inviscid hydrodynamic contribution while the second term is the Maxwell stress - for more details see Jackson [21, Chapter 6]. The boundary conditions on the channel walls are a no penetration condition for the hydrodynamics and zero vertical component of the electric field for the electrostatics (physically this models electrically insulating walls)

$$
\phi_{1 y}=V_{1 y}=0 \quad \text { at } \quad y=0, \quad \phi_{2 y}=V_{2 y}=0 \quad \text { at } \quad y=D_{u} .
$$

Far away from any interfacial disturbances, the electric field tends to its unperturbed value so that

$$
V_{j x} \rightarrow-E_{0}, \quad \text { as }|x| \rightarrow \infty, \quad j=1,2 .
$$

Variables are nondimensionalized by scaling lengths with $D$, fluid potentials with $D(g D)^{1 / 2}$, time with $(D / g)^{1 / 2}$, and voltages with $V_{0}$. Such choice of scalings retains gravitational effects in the model and allows one to switch surface tension and electric field effects on or off directly. The problem then has the following dimensionless groups:

$$
\rho=\frac{\rho_{2}}{\rho_{1}}, \quad \epsilon_{p}=\frac{\epsilon_{2}}{\epsilon_{1}}, \quad E_{b}=\frac{\epsilon_{1} V_{0}^{2}}{\rho_{1} g D^{3}}, \quad W_{e}=\frac{\sigma}{\rho_{1} g D^{2}}, \quad H_{0}=\frac{D_{u}}{D},
$$

which represent the density and permittivity ratios, an electric Weber number $E_{b}$

Copyright (C) by SIAM. Unauthorized reproduction of this article is prohibited. 
measuring the strength of the electric field, an inverse Weber number $W_{e}$ based on the velocity $(g D)^{1 / 2}$ (it is the ratio of surface tension forces to gravitational forces - this is also an inverse Bond number), and the dimensionless channel height $H_{0}$. In what follows, the same symbols are used for dimensionless quantities as before. Changing to dimensionless variables we find that (2.1) and boundary conditions (2.2) and (2.6) are unchanged, while (2.4) becomes

$$
\begin{gathered}
V_{1 x} H_{x}-V_{1 y}=\epsilon_{p}\left(V_{2 x} H_{x}-V_{2 y}\right) \quad \text { at } \quad y=H(x, t), \\
V_{1 x}+V_{1 y} H_{x}=V_{2 x}+V_{2 y} H_{x} \quad \text { at } \quad y=H(x, t) .
\end{gathered}
$$

The dynamic boundary condition at $y=H(x, t)$ is derived as follows. First, the $x$ and $y$-momentum equations of the Euler equations are written in terms of the flow potentials $\phi_{1,2}$ in each respective region. Then they are integrated with respect to $x$ and $y$, respectively, and evaluated at the interface in order to obtain an expression for the pressure jump across the interface in terms of $\phi_{1,2}$ and their derivatives. Using the normal stress balance (2.3) to eliminate the pressure jump yields the dynamic boundary condition, which involves electrical, surface tension, and gravitational effects (for more details see $[15,6]$ ), and yields

$$
\begin{aligned}
\phi_{1 t}+ & \frac{1}{2}\left(\phi_{1 x}\right)^{2}+\frac{1}{2}\left(\phi_{1 y}\right)^{2}-\rho\left(\phi_{2 t}+\frac{1}{2}\left(\phi_{2 x}\right)^{2}+\frac{1}{2}\left(\phi_{2 y}\right)^{2}\right)-(\rho-1) H \\
= & W_{e} \frac{H_{x x}}{\left(1+H_{x}^{2}\right)^{3 / 2}}-\frac{E_{b}}{2}\left(V_{1 x}^{2}-V_{1 y}^{2}\right) \frac{H_{x}^{2}-1}{1+H_{x}^{2}}+\frac{\epsilon_{p} E_{b}}{2}\left(V_{2 x}^{2}-V_{2 y}^{2}\right) \frac{H_{x}^{2}-1}{1+H_{x}^{2}} \\
& +2 E_{b} \frac{H_{x}}{1+H_{x}^{2}} V_{1 x} V_{1 y}-2 \epsilon_{p} E_{b} \frac{H_{x}}{1+H_{x}^{2}} V_{2 x} V_{2 y}+\bar{K}_{p},
\end{aligned}
$$

where the constant of integration $\bar{K}_{p}=-(\rho-1)-\frac{E_{b}}{2}\left(1-\epsilon_{p}\right)$ has been determined by evaluating (2.11) at the steady-state $H=1, V_{1,2}=x, \phi_{1,2}=0$. It can be seen from (2.11) that the electric field or surface tension contributions can be removed by selecting $E_{b}$ or $W_{e}$ to vanish, respectively. The linear stability was considered in [6], where it was shown that the system is susceptible to Rayleigh-Taylor instability when $\rho>1$. More importantly, it was shown that even in the absence of surface tension $\left(W_{e}=0\right)$, the electric field is capable of stabilizing the Rayleigh-Taylor instability for all wavenumbers. If surface tension is also present, the stability is enhanced further.

2.2. Nonlinear dynamics for thin upper layers. The nonlinear moving boundary problem posed in section 2.1 is not directly amenable to analysis, and we proceed by considering a physically relevant case when the upper layers are thin. A detailed derivation can be found in [6], but we sketch the derivation for completeness. If $\epsilon$ is the undisturbed thickness of the upper layer, we assume that interfacial waves have amplitudes of order $O(\epsilon)$ and order one wavelengths (i.e., of comparable length with the lower layer depth). Vertical scales are separated by introducing a new upper layer variable $\zeta$ given by $y=1+\epsilon-\epsilon \zeta$. The disturbed interface is written as $H(x, t)=1+\epsilon S(x, t)$, where $S(x, t)$ is to be found, and time is scaled according to $\tau=\epsilon^{1 / 2} t$ to keep nonlinearity in Bernoulli equation. The fluid and voltage potentials are expanded as

$$
\begin{array}{cl}
\phi_{1}=\epsilon^{1 / 2}\left(\phi_{1}^{(0)}+\epsilon^{2} \phi_{1}^{(2)}+\cdots\right), & \phi_{2}=\epsilon^{1 / 2}\left(\phi_{2}^{(0)}+\epsilon^{2} \phi_{2}^{(2)}+\cdots\right) \\
V_{1}=x+\epsilon V_{1}^{(1)}+\cdots, & V_{2}=x+\epsilon V_{2}^{(1)}+\cdots
\end{array}
$$

where the $x$ terms in (2.12) represent the background voltage potentials.

Copyright (C) by SIAM. Unauthorized reproduction of this article is prohibited. 
Solving the problem in the film region, we find $\phi_{2}^{(0)}=B(x, \tau)$ and $\phi_{2}^{(2)}=-\frac{\zeta^{2}}{2} B_{x x}$, where $B(x, \tau)$ is to be determined. The electric potential $V_{2}$ has the leading order solution $V_{2}^{(1)}=C(x, \tau)$ with $C(x, t)$ to be determined. The second kinematic condition (2.2) becomes

$$
S_{\tau}+\left((S-1) B_{x}\right)_{x}=0 .
$$

In the lower layer, the fluid potential leading order term reduces to $\phi_{1}^{(0)} \equiv$ const. This simplifies significantly the dynamic boundary condition (2.11), which after using electric field boundary conditions becomes, to the leading order,

$$
-\rho\left(\phi_{2 \tau}^{(0)}+\frac{1}{2}\left(\phi_{2 x}^{(0)}\right)^{2}\right)-(\rho-1) S=W_{e} S_{x x}+E_{b}\left(1-\epsilon_{p}\right) C_{x x} .
$$

Solving the harmonic problem for $V_{1}^{(1)}$ in Fourier space, we are able to express $C(x, \tau)$ in terms of $S(x, t)$ as

$$
\hat{C}=i\left(1-\epsilon_{p}\right)(\operatorname{coth} k) \hat{S}
$$

Introducing new variables $\eta(x, \tau)=1-S(x, \tau), u(x, \tau)=B_{x}(x, \tau)$, where $\eta$ represents the scaled upper layer thickness and $u$ the horizontal velocity at the interface and using $t$ instead of $\tau$, transforms (2.13)-(2.15) into the following nonlocal model system

$$
\begin{aligned}
\eta_{t}+(\eta u)_{x} & =0, \\
\rho\left(u_{t}+u u_{x}\right)-(\rho-1) \eta_{x} & =W_{e} \eta_{x x x}+\bar{E}_{b} \mathcal{L} \eta_{x x},
\end{aligned}
$$

where $\bar{E}_{b}=\left(1-\epsilon_{p}\right)^{2} E_{b} \geq 0$ and the linear operator $\mathcal{L}$ is known through its Fourier spectrum

$$
\widehat{\mathcal{L} \eta}=i(\operatorname{coth} k) \hat{\eta} \text {. }
$$

The system $(2.16)-(2.18)$ is solved as an initial value problem with periodic boundary conditions in $x, \eta(x+2 \pi, t)=\eta(x, t), u(x+2 \pi, t)=u(x, t)$. Numerical solutions are considered later; first, we present the linear aspects of the system.

3. Linear theory. We begin by considering the linear stability of the full problem stated in section 2.1. The dispersion relation has been given in [6], and we include it here for completeness in order to identify various limits. Linearizing about the exact solution $\mathbf{u}_{1,2}=\mathbf{0}, H=1, V_{1,2}=x$ (flat interface, quiescent flow, and uniform electric field) and introducing normal modes into the perturbations, i.e., writing $\phi_{1,2}(x, y, t)=\hat{\phi}_{1,2}(y) \mathrm{e}^{i k x+\omega t}+c . c$. , etc., provides a system of ODEs in $y$; these can be solved in closed form, and on use of the boundary conditions at the walls and the linearized interfacial conditions, the following dispersion relation is obtained:

$$
\begin{aligned}
\frac{\omega^{2}}{k}(\operatorname{coth} k-\rho & \left.\rho \frac{\cosh k-\tanh \left(k H_{0}\right) \sinh k}{\sinh k-\tanh \left(k H_{0}\right) \cosh k}\right)-(\rho-1)=-W_{e} k^{2} \\
& +\frac{\bar{E}_{b} k\left[\cosh k-\tanh \left(k H_{0}\right) \sinh k\right]}{-\left(1-\epsilon_{p}\right) \sinh k-\epsilon_{p} \tanh \left(k H_{0}\right) \cosh k+\tanh \left(k H_{0}\right) \frac{\sinh ^{2} k}{\cosh k}} .
\end{aligned}
$$

Surface tension and electric field effects enter through the first and second terms on the right-hand side of (3.1), respectively. When both of these terms are absent, the 
flow is unstable if $\rho>1$ as expected (the heavier fluid is on top). As a check, we note that (3.1) recovers the result in [28], once gravity and the effect of the upper fluid are removed along with the upper wall position sent to infinity, $H_{0} \rightarrow \infty$.

To better understand the stabilizing effect of the electric field, we take $H_{0}>1$ and consider (3.1) in the short-wave limit, $k \gg 1$, to find

$$
\omega^{2}=\frac{\rho-1}{\rho+1} k-W_{e} \frac{k^{3}}{\rho+1}-\frac{E_{b}\left(1-\epsilon_{p}\right)^{2}}{(\rho+1)\left(1+\epsilon_{p}\right)} k^{2}, \quad k \gg 1 .
$$

The electric field term is negative for all $k$ and hence provides an additional dispersive regularization to that of surface tension. In particular, if surface tension is absent $\left(W_{e}=0\right)$, we see from (3.2) that the electric field is capable of stabilizing short waves. This novel physical regularization of Rayleigh-Taylor instability was also found in Kelvin-Helmholtz instability [16]. Furthermore, a sufficiently large electric field can completely stabilize Rayleigh-Taylor instability at all wavenumbers, and this follows from a long wave limit of (3.1). In what follows, we analyze this limit in the context of our thin upper layer model. Writing $H_{0}=1+\epsilon$ with $0<\epsilon \ll 1$, expanding (3.1) in $\epsilon$, and taking into account the time rescaling $t \rightarrow \epsilon^{1 / 2} t$ that dictates the rescaling of $w \rightarrow \epsilon^{1 / 2} w$ yields to leading order

$$
\omega^{2} \rho=(\rho-1) k^{2}-W_{e} k^{4}-\bar{E}_{b} k^{3} \operatorname{coth} k
$$

which in the long-wave regime $(k \ll 1)$ reduces to

$$
\omega^{2} \rho=(\rho-1) k^{2}-W_{e} k^{4}-\bar{E}_{b} k^{2} .
$$

Equation (3.3) is, in fact, the dispersion relation of the model equations (2.16)-(2.18) and can be derived directly from those equations by linearizing about $\eta=1$ and $u=0$. The result (3.3) indicates that the nonlocal system (2.16)-(2.18) is dispersive if $\bar{E}_{b}$ is sufficiently large.

When surface tension and electric fields (if present) are weak, the interface can become unstable. Numerical solutions presented in the next section suggest that in this case the interface develops a touch up singularity, corresponding to $\eta \rightarrow 0$, after a finite time $t_{s}$ with the velocity $u$ blowing up at that time. Different terminal structures are found depending on whether $W_{e}$ is zero or not. In the former case, we must have $E_{b} \neq 0$ to ensure that the problem is not short-wave unstable. On the other hand, if $W_{e}$ and/or $E_{b}$ are sufficiently large to provide linearly stable solutions (according to (3.3), for instance), the solutions undergo persistent oscillations (typically quasiperiodic in time) for fairly general and moderately large initial conditions.

4. Numerical solutions to initial value problems. In this section we solve the evolution equations (2.16)-(2.18) subject to prescribed initial conditions on spatially periodic domains, and for various values of the dimensionless parameters $W_{e}, \bar{E}$, and $\rho$. We implement pseudospectral methods in space and a four-stage Runge-Kutta method in time (as in [36]). The solution is spectrally interpolated to increase spatial accuracy, when the amplitude of any of the last $20 \%$ of the modes exceeds a chosen tolerance level. The tolerance depends on the number of modes $N$, and typically increases as $N$ increases. In our simulations, $N$ varies from $2^{9}$ to $2^{15}$ and the tolerance level varies from $10^{-12}$ with $N=2^{9}$ to $10^{-5}$ with $N=2^{15}$. The time step is also adaptive and is decreased as $N$ increases. To maintain numerical stability, we require a time step $\Delta t$ to satisfy $k^{2} \Delta t<\nu$, where $\nu=10^{-3}$ and $k$ is the largest wave number retained in the calculations. The restriction on the time step comes from the linear

Copyright (C) by SIAM. Unauthorized reproduction of this article is prohibited. 

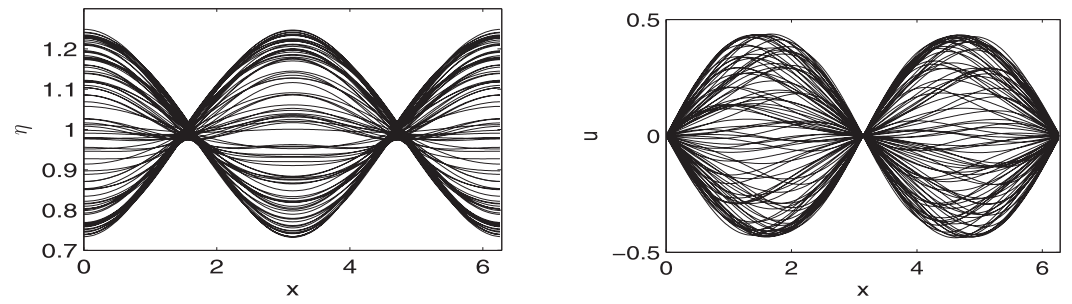

Fig. 2. Evolution of $\eta$ (left panel) and $u$ (right panel) with $W_{e}=1, \rho=3, \bar{E}_{b}=3$.
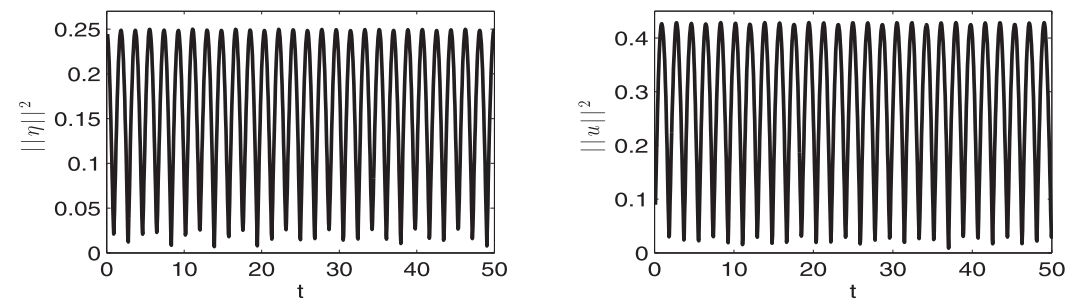
b.

dispersion relation for large wave numbers. For details, please see [36]. In addition, we use the smooth spectral filtering proposed by Hou and $\mathrm{Li}$ [19] in order to remove any aliasing errors and smooth the highest Fourier modes. We found that the filter suppresses high frequency instability and allows us to compute accurate solutions close to the singular time.

4.1. Linearly stable regime with surface tension and electric field present: Nonlinear dynamics. As seen from (3.3), sufficiently large values of $W_{e}$ and $\bar{E}_{b}$ render the flat state linearly stable. In this section we consider nonlinear perturbations that are outside the realm of linear theory but for linearly stabilizing values of $W_{e}$ and $\bar{E}_{b}$. We illustrate such dynamics through numerical solutions of (2.16)-(2.18) for $W_{e}=1, \rho=3, \bar{E}_{b}=3$, along with the initial condition

$$
\eta(x, 0)=1-\frac{1}{4} \cos x, \quad u(x, 0)=0 .
$$

Figure 2 shows the spatiotemporal evolution of $\eta(x, t)$ (left panel) and the corresponding horizontal velocity $u(x, t)$ (right panel) over a time interval of approximately 50 time units. The solutions are standing waves that in fact undergo quasi-periodic oscillations in time. At the nodes of $\eta$ (these are at $x=\pi / 2,3 \pi / 2$ ), the velocity has its maximum magnitude, whereas at the nodes of $u$ (at $x=0, \pi), \eta$ takes on its maximum values as expected physically. The corresponding energy norms of $\eta$ and $u$ are shown in Figure 3. We define the energy norm $\|f\|$ or $L_{2}$-norm of a function $f(x)$ with $x \in[0,2 \pi]$ as $\|f\|^{2}=\int_{0}^{2 \pi} f^{2} d x=\sum_{n=-\infty}^{\infty}\left|c_{n}\right|^{2} \approx \sum_{n=-N / 2+1}^{N / 2}\left|c_{n}\right|^{2}$, where $c_{n}$ are Fourier coefficients of $f(x)$, and $N$ is the number of Fourier coefficients we retain. The time oscillations in the energy norms are similar with the most energetic frequency being the same; the signals are, however, quasi-periodic (and different) — in both cases lower incommensurate frequencies enter to modulate the dynamics. Poincaré sections can be used to confirm torus-like dynamics - see, for example, [18] for such numerical constructions in a different problem. It is clear from these results that the solutions 

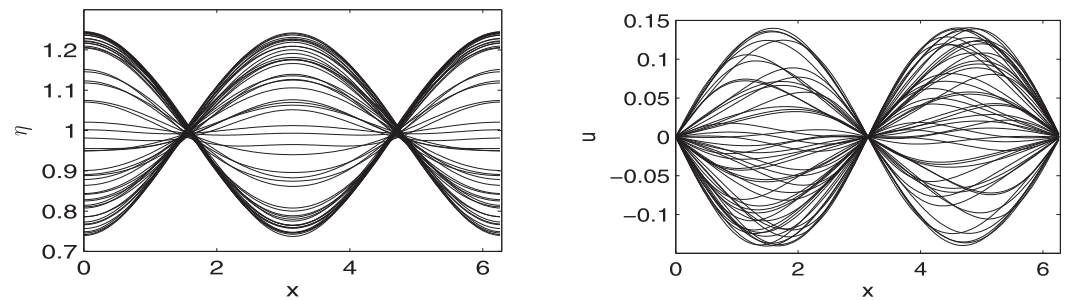

FIG. 4. Evolution of $\eta$ (left panel) and $u$ (right panel) with $W_{e}=0, \rho=2, \bar{E}_{b}=1$. Note that dynamics in this case is very similar to the case in Figure 2 when surface tension is present. As we show later, the structure of singular solutions with and without surface tension, however, is not the same.
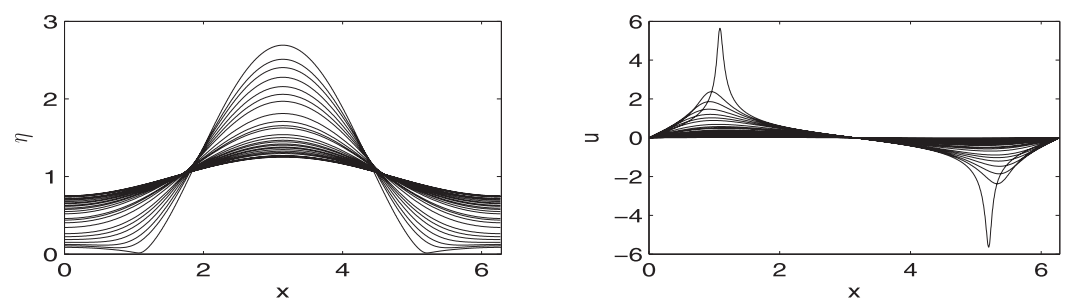

FIG. 5. Evolution of $\eta$ (left panel) and $u$ (right panel) with $W_{e}=1, \rho=4, \bar{E}_{b}=1$.

enter a persistent time oscillatory nonlinear motion that appears to be quasi-periodic. The absence of damping guarantees that these oscillations are self-sustaining and the solution remains smooth as long as the initial amplitude is not too large - in this example, it is a quarter of the undisturbed fluid layer depth.

We also note that similar behavior is found if either of $W_{e}$ or $\bar{E}_{b}$ is zero, and the other is sufficiently large. We include results for the case $W_{e}=0, \rho=2, \bar{E}_{b}=1$, i.e., when surface tension is absent and the stabilization of the Rayleigh-Taylor instability is completely due to the electric field. The spatiotemporal evolution of $\eta$ and $u$ are depicted in Figure 4, which is seen to be analogous to Figure 2.

4.2. Linearly unstable regime: Nonlinear evolution and finite-time singularities. When $W_{e}$ and $\bar{E}_{b}$ are chosen so that the flow is unstable to linear disturbances, the nonlinear dynamics are quite different and generically lead to singularity formation in finite time. The interface touches the wall, and the local fluid velocity becomes unbounded. There are two canonical cases: first when surface tension is present and $W_{e} \neq 0$ (with $\bar{E}_{b}$ arbitrary including $\bar{E}_{b}=0$ ), and second when surface tension is absent and $W_{e}=0$ but in the presence of electric field regularizing effects, $\bar{E}_{b} \neq 0$. In what follows, we illustrate the dynamics with sample numerical computations and in subsequent sections consider in detail the structure of the singularities in each canonical case.

We begin with surface tension and electric fields being present and solve numerically the initial value problem (with initial condition in (4.1)) with $W_{e}=1, \rho=4$, and $\bar{E}_{b}=1$. The evolution of the interfacial shape $\eta$ and corresponding velocity $u$ is given in Figure 5. The results strongly indicate that the interface touches up in finite time, which corresponds to $\eta$ approaching 0 (there are two symmetrically placed touch-up points), and the corresponding velocity in the vicinity of touch-up grows unbounded with fluid being pushed into the main central drop. The velocity is negative in the vicinity of the rightmost touch-up point and positive near the left one. Additional 

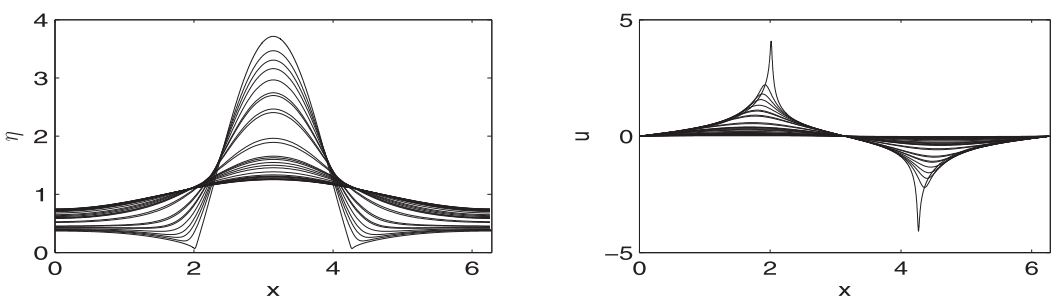

FIG. 6. Evolution of $\eta$ (left panel) and $u$ (right panel) with $W_{e}=0, \rho=3, \bar{E}_{b}=1$.

evidence is provided by the evolution of the energy norms of both $\eta$ and $u$ (graphs are not shown), which, unlike the linearly stable cases, do not oscillate quasi-periodically but instead they grow monotonically with a suggestion that they become infinite in finite time. More detailed analysis of the singularities is presented later.

The next set of results removes surface tension and retains electric fields. The parameters used are $W_{e}=0, \rho=3$, and $\bar{E}_{b}=1$. The dispersion relation (3.3) indicates that the short-wave regularization is different depending on whether $W_{e}$ is zero or not, and we expect the nonlinear dynamics and, in particular, the structure of terminal singular structures to also be different. Before analyzing such aspects, we illustrate the dynamics in Figure 6 that depict the spatiotemporal evolution of $\eta$ and $u$. We also investigated the evolution of the corresponding energy norms. The overall qualitative features of the results are similar to those having $W_{e} \neq 0$, but we note the presence of sharper features in the touch-up and velocity profiles when $W_{e}=0$. It is important, therefore, to quantify these differences mathematically by considering the solutions near the singularity in more detail. We note that the scaled interfacial profiles $\eta$ in Figures 5 and 6 remain bounded throughout their evolution. Given that the position of the interface is $S(x, t)=1+\epsilon-\epsilon \eta(x, t)$, we conclude that the interface does not penetrate into the lower region 1 by more than a distance of order $\epsilon$, and so we do not obtain penetrating fingers of length comparable to the channel height.

5. Finite-time singularities and self-similar solutions. The numerical results of section 4 indicate that the evolution equations (2.16)-(2.18) possess singularities with the interface vanishing at a point(s) and the local velocity blowing up there after a finite time. We are interested in quantifying such terminal states and establishing the dominant solutions at breakup by performing a local analysis and constructing self-similar solutions. The similarity solutions that emerge are of the second kind and involve an exponent that cannot be fixed by an order-of-magnitude analysis but must be determined through careful numerical experiments. In what follows, we address the description of local structures at breakup. We derive selfsimilar scalings and investigate the spatial and temporal structure of the singularity in the model given by (2.16)-(2.18). The scalings for the case when surface tension is present are different from the case without it since the leading order contributions to the singular solutions are different. Details are considered next.

5.1. Singularities and self-similar solutions when surface tension is present, $\boldsymbol{W}_{\boldsymbol{e}} \neq \mathbf{0}$. Consider the system (2.16)-(2.18) and assume that the interface vanishes at a point(s) $x=x_{s}$ and the velocity blows up there after a finite time, $t_{s}$, say. We look for self-similar solutions for $\tau=t_{s}-t \ll 1$ by writing

$$
\eta(t, x)=\tau^{a} f(\xi), \quad u(t, x)=\tau^{b} g(\xi), \quad \xi=\frac{x-x_{s}}{\tau^{\alpha}},
$$

Copyright @ $\odot$ by SIAM. Unauthorized reproduction of this article is prohibited. 
where the constants $a>0, b<0$ (in accordance with the numerical evidence of section 4), and $\alpha$ are to be determined. Balancing terms in (2.16) as $\tau \rightarrow 0$ gives $u \sim \tau^{\alpha-1}$. A balance of terms in (2.17) shows that surface tension dominates over the gravity and electric field terms at touch-up, and to leading order we obtain $\frac{u}{\tau} \sim$ $\frac{u^{2}}{\left|x-x_{s}\right|} \sim \frac{\eta}{\left|x-x_{s}\right|^{3}}$, which in turn leads to $\eta \sim \tau^{4 \alpha-2}$ and $u \sim \tau^{\alpha-1}$. Hence, the similarity solutions have the following form:

$$
\eta=\left(t_{s}-t\right)^{4 \alpha-2} f(\xi), \quad u=\left(t_{s}-t\right)^{\alpha-1} g(\xi), \quad \xi=\frac{x-x_{s}}{\left(t_{s}-t\right)^{\alpha}}
$$

For the interface to touch up with $\eta \rightarrow 0$ and $u$ blow up, we must have $4 \alpha-2>0$ and $\alpha-1<0$, i.e., $1 / 2<\alpha<1$ is necessary.

Substituting the ansatz (5.2) into (2.16)-(2.18) and retaining leading order terms gives the following equations for the scaling functions $f$ and $g$ :

$$
-(4 \alpha-2) f+\alpha \xi f^{\prime}+(f g)^{\prime}=0, \quad-(\alpha-1) g+\alpha \xi g^{\prime}+g g^{\prime}=\frac{W_{e}}{\rho} f^{\prime \prime \prime} .
$$

The behavior of the scaling functions $f$ and $g$ for large $\xi$ can be found by noticing that at leading order (5.3) yields

$$
-(4 \alpha-2) f+\alpha \xi f^{\prime}=0, \quad-(\alpha-1) g+\alpha \xi g^{\prime}=0 \quad \text { for } \quad|\xi| \gg 1
$$

with solutions

$$
f \sim|\xi|^{4-\frac{2}{\alpha}}, \quad g \sim|\xi|^{1-\frac{1}{\alpha}} \quad \text { as } \quad|\xi| \gg 1 .
$$

Note that these scalings could have been anticipated by using the fact that the solution far from the singular point is quasi-static, to leading order, and hence the behavior of $f$ and $g$ must have the algebraic forms (5.5) to cancel out the time dependent scaling factors $\tau^{4 \alpha-2}$ and $\tau^{\alpha-1}$, respectively. For details, see section 5.1.3.

To numerically validate the self-similar scalings, we observe that according to (5.2), the maximum curvature $\left|\eta_{x x}\right|$ and the maximum of $u^{2}$ over the $2 \pi$-periodic domain have the asymptotic behavior

$$
\max _{0<x \leq 2 \pi}\left|\eta_{x x}\right| \sim\left(t_{s}-t\right)^{2 \alpha-2}, \quad \max _{0<x \leq 2 \pi} u^{2} \sim\left(t_{s}-t\right)^{2 \alpha-2},
$$

and these quantities are therefore proportional to each other. This is useful since plots of $\max \left|\eta_{x x}\right|$ against $\max u^{2}$, with time as a parameter, are expected to give a straight line as the singularity is approached. Numerical results are shown in Figure 7 for two sets of parameters: (i) $W_{e}=1, \rho=4, \bar{E}_{b}=1$ and (ii) $W_{e}=3, \rho=5, \bar{E}_{b}=0$. We see clearly that in both cases (whether the electric field is present or not) the plots produce straight lines and hence corroborate the self-similar scalings (5.2).

Plots of other quantities that are of the same order have also been tested with equally good results. The value of $\alpha$ remains undetermined, however, and we turn our attention to this next.

We have numerically estimated $\alpha$ using three methods. The first two methods are based on the temporal structure of singularity: one method relies on knowing $t_{s}$ accurately (this again must be estimated from the numerical solutions), and the other does not require $t_{s}$. The third method, described in section 5.1.3, allows one to estimate $\alpha$ by analyzing the spatial structure of singularity. In what follows, we present these approaches and compare their performance. 

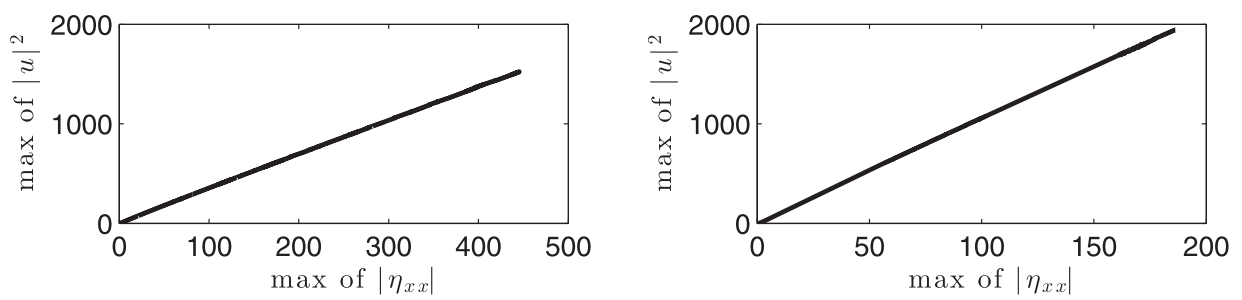

FIG. 7. Evolution of $\max$ of $\left|\eta_{x x}\right|$ vs. max of $|u|^{2}$ with $t$ being a parameter when $W_{e} \neq 0$. Left panel: $W_{e}=1, \rho=4, \bar{E}_{b}=1$. Right panel: $W_{e}=3, \rho=5, \bar{E}_{b}=0$.
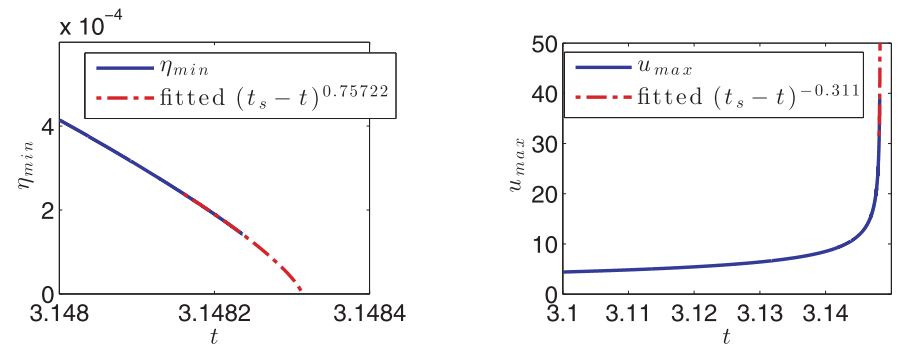

FIG. 8. Left panel: Graph of $\eta_{\min }$ with superimposed fitted curve $\left(t_{s}-t\right)^{0.75722}$ in the case $W_{e}=1, \rho=4, \bar{E}_{b}=1$. We find $t_{s}=3.148313$. Right panel: $u_{\max }$ and fitted curve $\left(t_{s}-t\right)^{-0.311}$.

5.1.1. Estimation of $t_{s}$ and calculation of $\alpha$. According to (5.2), the minimum layer thickness and corresponding maximum velocity have the form

$$
\eta_{\min } \sim\left(t_{s}-t\right)^{4 \alpha-2}, \quad u_{\max } \sim\left(t_{s}-t\right)^{\alpha-1} .
$$

In order to estimate the value of $\alpha$, we monitor $\eta_{\min }(t)$ and $u_{\max }(t)$ as a singularity is encountered and apply data fitting techniques to find $\alpha$ and $t_{s}$. For accurate results, we calculate $\eta_{\min }$ and $u_{\max }$ at a given time by performing polynomial interpolation (typically quadratic) on the grid in the vicinity of the local minimum or maximum. Our computations for the case $W_{e}=1, \rho=4, \bar{E}_{b}=1$ show that $\eta_{\text {min }}$ approaches zero as $\left(t_{s}-t\right)^{0.75722}$ as $t$ approaches $t_{s} \approx 3.148313$. To illustrate how the exponents are extracted, in Figure 8 we present plots of the evolution of $\eta_{\min }$ and $u_{\max }$ (solid curves) and superimpose best fit curves (dash-dot curves). The fits are obtained by starting with the asymptotic behavior (5.6) and noting, for example, that for the linear relation $\ln \eta_{\min }=C+(4 \alpha-2) \ln \left(t_{s}-t\right)$ (an analogous expression holds for $\ln u_{\text {max }}$ ), the least squares method can be used to estimate the constant $C$, the slope $(4 \alpha-2)$ and the singular time $t_{s}$. We find $t_{s} \approx 3.148313$ and $\alpha \approx 0.6893$, and these are fully consistent with the corresponding results from the least squares fit of the $u_{\max }$ data. The latter yield $u_{\max } \approx\left(t_{s}-t\right)^{-0.30902}$, and hence $\alpha \approx 0.6910$. Both estimates of $\alpha$ agree well. The difference is $1.7 \times 10^{-3}$, or less than $0.25 \%$. For completeness, in Figure 9 we include the logarithmic plots of the evolution of $\eta_{\min }$ and $u_{\max }$ together with superimposed least squares fits used to estimate the slopes near the singularity. For comparison, we also plot $\eta_{\min } \approx\left(t_{s}-t\right)^{0.756}$ and $u_{\max } \approx\left(t_{s}-t\right)^{-0.311}$, whose exponents are obtained using an alternative method based on the temporal structure of singularity as well but it allows one to estimate $\alpha$ without a priori knowledge of $t_{s}$. The details of the method are given in section 5.1.2.

We have also confirmed the similarity scalings (5.2) when the electric field is not present. In particular, we set $\bar{E}_{b}=0$ and carried out two runs for $W_{e}=1, \rho=3$, and 

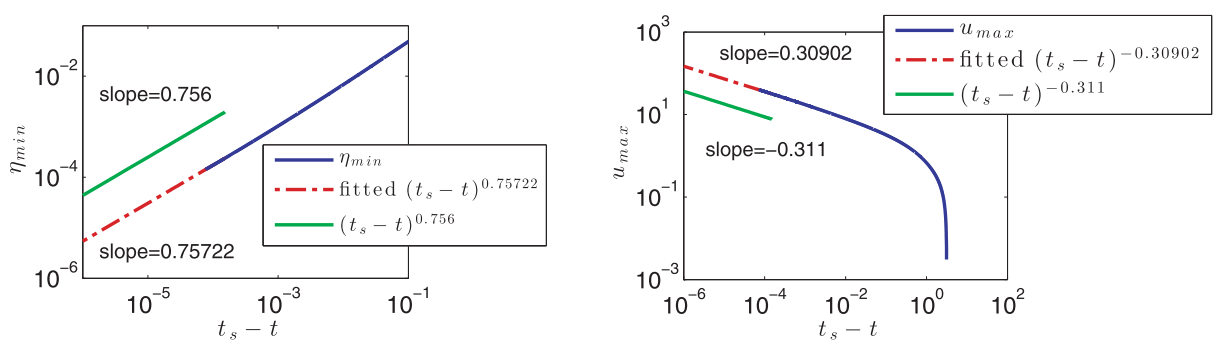

FIG. 9. Case $W_{e}=1, \rho=4, \bar{E}_{b}=1$. Left panel: Log-log plot of $\eta_{\min }$ with superimposed fitted curve $\left(t_{s}-t\right)^{0.75722}$ and curve $\left(t_{s}-t\right)^{0.756}$. The latter exponent is obtained using an alternative approach described in section 5.1.2. Right panel: Log-log plot of $u_{\max }$ with respective superimposed curves.

TABLE 1

Comparison of approximations of $\alpha$ obtained from $\eta_{\min }$ and $u_{\max }$ for different sets of $W_{e}, \rho$ and $\bar{E}_{b}$, starting from the initial condition $\eta(x, 0)=1-0.25 \cos x, u(x, 0)=0$. The simulations were terminated when $\eta_{\min }(t)=10^{-3}$.

\begin{tabular}{|c|c|c|c|c|c|}
\hline$W_{e}$ & $\rho$ & $\bar{E}_{b}$ & $\alpha$ from $\eta_{\min }$ & $\alpha$ from $u_{\max }$ & $t_{s}$ \\
\hline 1 & 4 & 1 & 0.6893 & 0.6910 & 3.148313 \\
\hline 1 & 3 & 0 & 0.6898 & 0.6903 & 2.671165 \\
\hline 3 & 5 & 0 & 0.6891 & 0.6931 & 2.526857 \\
\hline
\end{tabular}

$W_{e}=3, \rho=5$. In both sets of computations, we estimated values of $\alpha$ independently by fitting the $\eta_{\min }$ and $u_{\max }$ evolution as described above. For the case $W_{e}=1, \rho=3$, we find $\alpha \approx 0.6898$ and $\alpha \approx 0.6903$, respectively, while for $W_{e}=3, \rho=5$, we obtain $\alpha \approx 0.6891$ and $\alpha \approx 0.6931$, respectively. The difference between the obtained values of $\alpha$ is at most $4 \times 10^{-3}$ and less than $1 \%$. The results are summarized in Table 1 , and agreement is very good.

5.1.2. Calculation of $\alpha$ without estimation of $\boldsymbol{t}_{\boldsymbol{s}}$. In this section we present an alternative way to estimate the conjectured scaling exponents found from the orderof-magnitudes arguments. This method has an advantage that one does not need to know the numerical estimate of $t_{s}$ explicitly, and hence provides an independent test of the self-similar ansatz. The self-similar solutions (5.2) are asymptotically valid in the limit $0<t_{s}-t \ll 1$, and hence we can express the quantities $M_{1}(t)=\ln \left(u_{\max }\right)$, $M_{2}(t)=\ln \left(\eta_{\min }\right)$ and $M_{3}(t)=\ln \left(\left|u_{x}\right|_{\max }\right)$ as

$$
\begin{gathered}
M_{1}(t)=(\alpha-1) \ln \left(t_{s}-t\right)+K_{1}(t), \quad M_{2}(t)=(4 \alpha-2) \ln \left(t_{s}-t\right)+K_{2}(t), \\
M_{3}(t)=-\ln \left(t_{s}-t\right)+K_{3}(t),
\end{gathered}
$$

where $K_{1,2,3}(t)$ are corrections that diminish in size as $t \rightarrow t_{s}-$. Forming the ratios of these functions yields

$$
\lim _{t \rightarrow t_{s}-} \frac{M_{1}}{M_{3}}=1-\alpha, \quad \lim _{t \rightarrow t_{s}-} \frac{M_{2}}{M_{3}}=2-4 \alpha, \quad \lim _{t \rightarrow t_{s}-} \frac{M_{1}}{M_{2}}=\frac{\alpha-1}{4 \alpha-2} .
$$

It follows (by solving for $\alpha$ in each of the ratios in (5.7)) that the quantities $\phi_{13}(t)=$ $1-\frac{M_{1}(t)}{M_{3}(t)}, \phi_{23}(t)=\frac{1}{4}\left(2-\frac{M_{2}(t)}{M_{3}(t)}\right), \phi_{12}(t)=\left(2 \frac{M_{1}(t)}{M_{2}(t)}-1\right) /\left(4 \frac{M_{1}(t)}{M_{2}(t)}-1\right)$ should all converge 

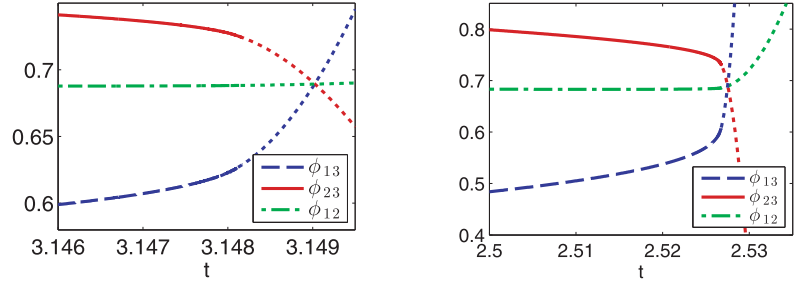

FIG. 10. Evolution of functions $\phi_{13}(t), \phi_{23}(t)$, and $\phi_{12}(t)$ as $t$ approaches $t_{s}^{-}$. Thicker curves represent numerical data, while thinner dotted curves are obtained from fitting to a quadratic polynomial. Left panel: Case $W_{e}=1, \rho=4, \bar{E}_{b}=1$. Extrapolated curves intersect at $t \approx 3.149$. Right panel: Case $W_{e}=3, \rho=5, \bar{E}_{b}=0$. Curves intersect at $t=2.5275$. In both cases, the vertical coordinate of the point of intersection of extrapolated curves is $\alpha \approx 0.689$.

to $\alpha$ in the limit, i.e., $\lim _{t \rightarrow t_{s}-} \phi_{13}=\lim _{t \rightarrow t_{s}-} \phi_{23}=\lim _{t \rightarrow t_{s}-} \phi_{12}=\alpha$. The evolution of these three functions $\phi_{13}, \phi_{23}$, and $\phi_{12}$ is shown in Figure 10 for two runs with parameters $W_{e}=1, \rho=4, \bar{E}_{b}=1$ (left panel) and $W_{e}=3, \rho=5, \bar{E}_{b}=0$ (right panel), respectively. The latter set of parameters has the electric field switched off. The curves beyond the times of the simulation (indicated by dotted curves) are obtained by using a quadratic polynomial extrapolation to find their point of intersection. For the results corresponding to the first set of parameters shown in the left panel of Figure 10, the prediction is a time of intersection at $t=t_{s} \approx 3.149$ (the abscissa) with a value of $\alpha \approx 0.689$ (the corresponding ordinate). For the second set of parameters (right panel) of Figure 10, the point of intersection is found to be $t=t_{s} \approx 2.5275$ with the corresponding ordinate giving an independently estimated value of $\alpha \approx 0.689$. We see that agreement between the two values of $\alpha$ is excellent, and note that agreement is also very good with the values of $\alpha$ found in section 5.1.1 with an alternative method. The method of this section will be used in the rest of the paper since it involves less fitting and does not rely on accurate a priori estimates of $t_{s}$. The horizontal coordinate (the time $t_{s}$ ) of the point of intersection of these three curves depends on the fitting functions used, but the vertical coordinate (the value of $\alpha$ ) can be obtained very robustly, primarily because the function $\phi_{12}$ is essentially constant for times close to $t_{s}$. In fact, it is sufficient to monitor the evolution of $\phi_{12}$ alone to obtain a good estimate for the value of $\alpha$, but for completeness we have included the other two ratios also. We can conclude, therefore, that $\alpha$ can be found more accurately using the present method, whereas it is preferable to estimate the value of $t_{s}$ using extrapolation of $\eta_{\min }$ to 0 , as was done in section 5.1.1.

5.1.3. Spatial structure of singularity in the presence of surface tension, $\boldsymbol{W}_{\boldsymbol{e}} \neq \mathbf{0}$. To obtain the leading order spatial structure of the singularity (and hence predict theoretically the form of the spectrum of the solutions as the singular time is approached), it is sufficient to consider the asymptotic forms of the self-similar solutions of (5.3) for large $|\xi|$. This in turn provides a third independent way of estimating the scaling parameter $\alpha$, as we describe next.

The leading order behavior (5.5) can also be seen by noting that away from the touch-up region, the solutions are quasi-static and, hence, independent of $\tau$, to leading order (see $[25,26]$ for a related problem). Assuming the asymptotic forms

$$
f(\xi) \sim|\xi|^{p}, \quad g(\xi) \sim|\xi|^{-q}, \quad p, q>0
$$

and expressing the solutions in terms of the outer variable $x-x_{s}$ (see (5.1)) yields

$$
\eta \sim \tau^{4 \alpha-2}|\xi|^{p}=\tau^{4 \alpha-2-\alpha p}\left|x-x_{s}\right|^{p}, \quad u \sim \tau^{\alpha-1}|\xi|^{-q}=\tau^{\alpha-1+\alpha q}\left|x-x_{s}\right|^{-q} .
$$



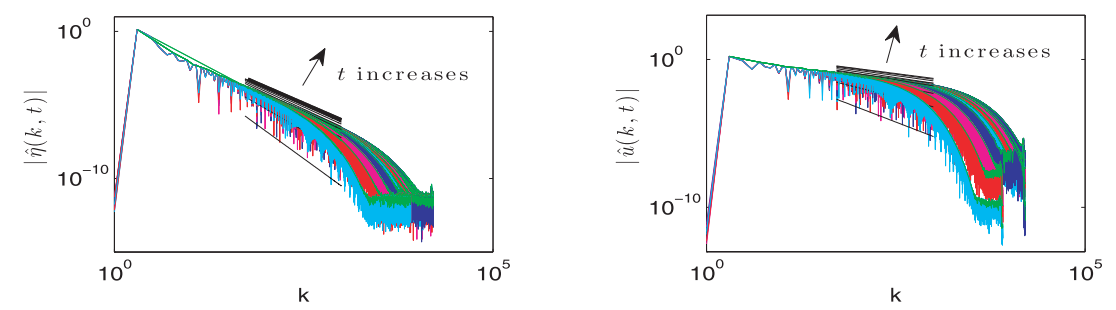

FIG. 11. Log-log plots of $|\hat{\eta}(k, t)|$ (left panel) and $|\hat{u}(k, t)|$ (right panel) at times close to ts for the case $W_{e}=1, \rho=4, \bar{E}_{b}=1$. Black straight lines are results of a fit of magnitudes of Fourier coefficients to linear functions of $\ln k$ to estimate the decay rates $\beta$ and $\gamma$, respectively.

Solutions (5.9) are independent of $\tau$ if $p=4-\frac{2}{\alpha}, q=\frac{1-\alpha}{\alpha}$, in complete agreement with (5.5). It follows that the leading order singular behavior of the solutions in terms of the outer physical variable $x$ (this is what is seen in the computations) takes the form

$$
\eta(t, x) \sim\left|x-x_{s}\right|^{p}, \quad u(t, x) \sim\left|x-x_{s}\right|^{-q}, \quad \text { as } \quad\left|x-x_{s}\right| \ll 1 .
$$

We will use computations to estimate $p$ and $q$ according to the predictions (5.10), and then in turn obtain two numerical estimates for $\alpha$ given by

$$
\alpha \sim \frac{2}{4-p}, \quad \alpha \sim \frac{1}{1+q}
$$

Note that we also have $p=2(1-q)$, and this provides an additional accuracy check on the numerics and the emergence of self-similar structures.

To extract information about the spatial structure of our singular solutions, we consider the asymptotic behavior of the Fourier coefficients as the singular time is approached. For a function

$$
f(x, t) \approx\left(x-\left(x_{0}+i \delta(t)\right)\right)^{\beta(t)-1}
$$

that has a branch point of order $\beta(t)-1>-1$ at $x_{0}+i \delta(t)$, the $k \gg 1$ behavior of the Fourier coefficients follows the Laplace formula [9, p. 255]

$$
|\hat{f}(k, t)| \sim k^{-\beta(t)} \mathrm{e}^{-k \delta(t)}
$$

As time evolves, the singularity traces out a curve in the complex plane [34], and the vanishing of $\delta$ at $t=t_{s}$ corresponds to the loss of analyticity for real $x$ at this time. Since

$$
\ln |\hat{f}(k, t)| \sim-\beta(t) \ln k-\delta(t) k,
$$

we can estimate $\beta(t)$ and $\delta(t)$ by log-log or log-linear plots, respectively, utilizing the middle part of the spectrum for the former and the large $k$ part of the spectrum for the latter-[34]. The evolution of such log-log spectral plots at times close to $t_{s}$ for the case $W_{e}=1, \rho=4, \bar{E}_{b}=1$ is plotted in Figure 11. It can be seen that the Fourier spectra 

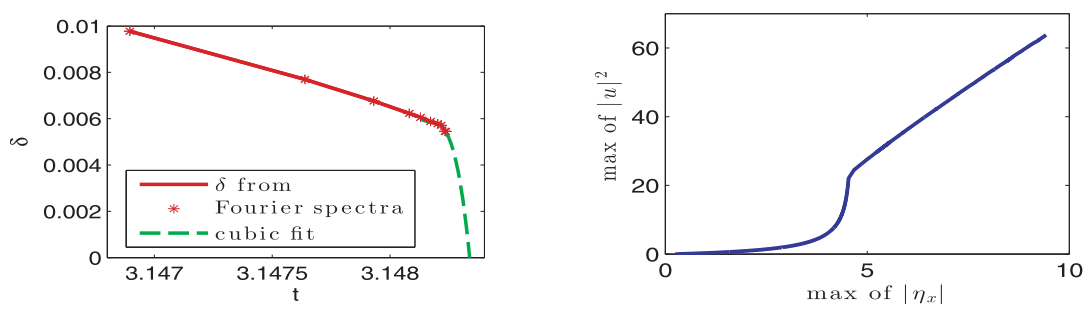

FIG. 12. Left panel: Evolution of $\delta$ in the case $W_{e}=1, \rho=4, \bar{E}_{b}=1$. The data at times close to $t_{s}$ were fit to a cubic polynomial and then extrapolated to find $t_{s}=3.148338975$ when $\delta=0$. Right panel: Evolution of $\max _{0 \leq x<2 \pi}\left\{u^{2}\right\}$ versus $\max _{0 \leq x<2 \pi}\left\{\left|\eta_{x}\right|\right\}$ in the case $W_{e}=0, \rho=3$, $\bar{E}_{b}=1$.

do not decay monotonically, and this suggests that there is a modulation caused by the presence of additional singularities that interfere with each other as found in different problems, e.g., [34, 29]. Due to this lack of monotonicity, we estimated slopes of the spectra by using the envelope formed by local maxima of the Fourier coefficients as shown by the straight lines just above the plots. The middle part of the spectrum $\left[k_{\text {start }}, k_{\text {end }}\right]$ is used with $k_{\text {start }}$ not too small and $k_{\text {end }}$ not too large; for example, for the results in Figure 11, the number of modes is $N=2^{14}=16384$ and typical appropriate values for the fit are $k_{\text {start }}=50$ and $k_{\text {end }}=1000$. For $t$ close to $t_{s}$, the spectra of $\eta$ and $u$ can be written as (see (5.12) and (5.13))

$$
\ln |\hat{\eta}| \sim-\beta(t) \ln k \sim-(p+1) \ln k, \quad \ln |\hat{u}| \sim-\gamma(t) \ln k \sim(q-1) \ln k,
$$

where $k \in\left[k_{\text {start }}, k_{\text {end }}\right]$. Beginning with $\eta, \beta(t)$ is estimated as described above for numerous times $t$ close to $t_{s}$. These estimates of $\beta$ are then fitted to a cubic polynomial and then extrapolated to $t_{s}=3.148313$ (this was estimated in section 5.1.1) to provide $\beta\left(t_{s}\right)=\beta_{s} \approx 2.1073$ (i.e., $p \approx 1.1073$ ), and this in turn enables the estimation of $\alpha \approx 0.69138$ from the first of relations (5.11). Note that even though the values of $\beta(t)$ appear to lie on a straight line, we found it better to fit them to a cubic polynomial to capture their dynamics at times close to $t_{s}$. In a similar way, the Fourier coefficients $\hat{u}$ are used to estimate $\gamma(t)$ as $t$ approaches $t_{s}$. Extrapolating to $t_{s}$, we obtain $\gamma_{s} \approx 0.53988$ (i.e., $q \approx 0.46012$ ), and, hence, $\alpha \approx 0.68487$ by the second relation in (5.11). We also mention that we investigated the sensitivity of computing $\beta_{s}, \gamma_{s}$, and, hence, $\alpha$, on the choice of $k_{\text {start }}, k_{\text {end }}$ and the order of fitting polynomials (up to the third order). As expected, small values of $k_{\text {start }}$, e.g., $k_{\text {start }}<20$, and large values of $k_{\text {end }}$, e.g., $k_{\text {end }}>2000$, do not provide accurate results. In addition, fitting to second or third order polynomials provides more accurate results than with a linear fit - we believe that this is due to a better capture of the nonlinear dynamics of the decay rates as $t$ approaches $t_{s}$.

For completeness, in the left panel of Figure 12 we also present the evolution of $\delta(t)$ as $t$ approaches $t_{s}$. As expected, $\delta(t) \rightarrow 0$ as $t \rightarrow t_{s}$, suggesting the loss of analyticity at $t_{s}$. The values of $\delta$ were estimated by considering the form (5.14) and producing log-linear plots as $k$ becomes large in order to obtain a straight line, to leading order, with slope $-\delta$. The values of $\delta$ for times close to $t_{s}$ are fit to a cubic polynomial and then extrapolated to find the time $t_{s}$ when $\delta=0$ - the extrapolating polynomial is shown by the dashed line. We find $t_{s} \approx 3.148339$, which agrees very well with the singular time computed previously using other approaches. 
In summary, then, the forms (5.10) along with the estimates $p \approx 1.1$ and $q \approx 0.46$ indicate that as touch-up takes place, we have, to leading order,

$$
\eta \sim\left|x-x_{s}\right|^{1.1}, \quad u \sim\left|x-x_{s}\right|^{-0.46} .
$$

This implies that the interface touches the wall with bounded slope but with an infinite curvature ( $\eta_{x x}$ becomes unbounded). At the same time, however, the corresponding horizontal velocity becomes singular as touch-up takes place, due to the squeezing of fluid away from the touch-up region. The long-wave models are no longer valid at touch-up and should be viewed as providing an outer picture of the dynamics - as shorter length-scales develop, all terms in the Euler equations must enter to provide a new asymptotic regime. Such analysis is left for future work.

5.2. Singularities and self-similar solutions in the absence of surface tension but with electric fields present: $W_{e}=0, E_{b} \neq 0$. In the absence of surface tension, a touch-up singularity still occurs (as indicated in Figure 6, for example), but the scalings are different since the highest derivative in the system (2.16)-(2.18) is due to the nonlocal term $\mathcal{L} \eta_{x x}$ rather than the capillary term $\eta_{x x x}$. The key in balancing terms as a singularity is approached and as spatial length scales shorten follows from the observation that short scales in real space correspond to large values of the wavenumber $k$ in Fourier space. Hence, the large $k$ behavior of $\widehat{\mathcal{L} \eta_{x x}}$ is relevant and we see from the definition (2.18) that

$$
\widehat{\mathcal{L} \eta_{x x}}=-i k^{2} \operatorname{coth} k \hat{\eta}(k) \sim-i \operatorname{sgn}(k) k^{2} \hat{\eta}(k)=-\widehat{\mathcal{H}\left[\eta_{x x}\right]},
$$

where we have introduced the Hilbert transform and used its properties and symbol in Fourier space,

$$
\mathcal{H}[\chi(x)]=\frac{1}{\pi} \int_{-\infty}^{\infty} \frac{\chi(y)}{x-y} d y, \quad \widehat{\mathcal{H}[\chi(x)]}=-i \operatorname{sgn}(k) \hat{\chi}(k) .
$$

Proceeding as in section 5.1, we balance terms in (2.16)-(2.18) as a singularity is approached, and assuming that the local length scale of the touch-up region is of order $\left(t_{s}-t\right)^{\alpha}$, the following scalings are suggested:

$$
\eta=\left(t_{s}-t\right)^{3 \alpha-2} f(\zeta), \quad u=\left(t_{s}-t\right)^{\alpha-1} g(\zeta), \quad \zeta=\frac{x-x_{s}}{\left(t_{s}-t\right)^{\alpha}} .
$$

Since the interface $\eta$ touches up with $\eta \rightarrow 0$ and $u$ blows up as $t \rightarrow t_{s^{-}}$, we have $3 \alpha-2>0, \alpha<1$, and hence the bounds $2 / 3<\alpha<1$. As in the $W_{e} \neq 0$ case, the exponent $\alpha$ is left undetermined since the self-similar solutions are again of the second kind. We will determine its value numerically and also confirm the self-similar structures posed by (5.19).

Substituting the ansatz (5.19) into the governing equations (2.16)-(2.18) and using the property (5.17), we obtain the following similarity equations for the scaling functions $f$ and $g$ :

$$
-(3 \alpha-2) f+\alpha \xi f^{\prime}+(f g)^{\prime}=0, \quad-(\alpha-1) g+\alpha \xi g^{\prime}+g g^{\prime}=-\frac{\bar{E}_{b}}{\rho} \mathcal{H}\left(f^{\prime \prime}\right) .
$$

Note that the gravity term has been dropped since it is asymptotically small compared to the terms retained. As in the nonzero surface tension case, we can find the behavior of $f$ and $g$ for large $|\zeta|$,

$$
f \sim|\xi|^{3-\frac{2}{\alpha}}, \quad g \sim|\xi|^{1-\frac{1}{\alpha}} \quad \text { for } \quad|\xi| \rightarrow \infty,
$$

Copyright @ $\odot$ by SIAM. Unauthorized reproduction of this article is prohibited. 

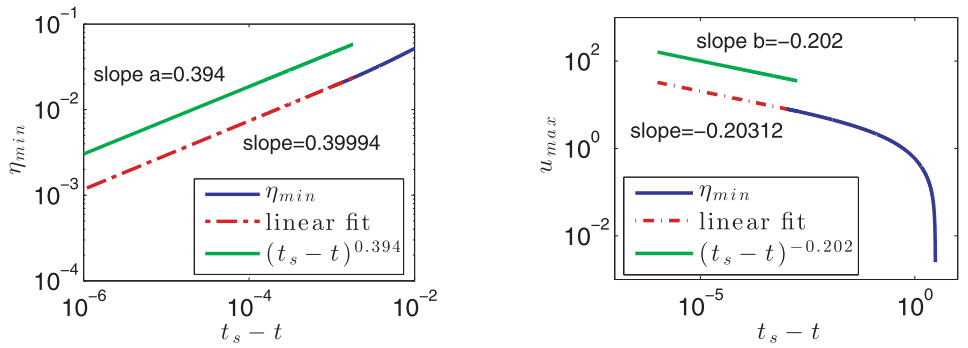

FIG. 13. Numerical results for $W_{e}=0, \rho=3, \bar{E}_{b}=1$ for $t$ close to $t_{s}=3.0333$. Left panel: Log-log plots of $\eta_{\min }$ versus $t_{s}-t$ with superimposed fitted function $\left(t_{s}-t\right)^{0.39994}$ and function $\left(t_{s}-t\right)^{0.394}$. The exponent 0.394 is obtained using a method for capturing temporal structure of singularity without a priori knowledge of $t_{s}$. Right panel: $u_{\max }$ and corresponding superimposed fitted function $\left(t_{s}-t\right)^{-0.20312}$ and the function $\left(t_{s}-t\right)^{-0.202}$ found without a priori knowledge of $t_{s}$.

as well as the corresponding spatial structure of the singularity suggested by the analysis,

$$
\eta \sim\left|x-x_{s}\right|^{\frac{3 \alpha-2}{\alpha}}, \quad u \sim\left|x_{s}-x\right|^{\frac{\alpha-1}{\alpha}} .
$$

To validate the similarity assumptions when $t$ is close to $t_{s}$, we note that

$$
\max _{0 \leq x<2 \pi}\left\{\left|\eta_{x}\right|\right\} \sim \tau^{2 \alpha-2}, \quad \max _{0 \leq x<2 \pi}\left\{u^{2}\right\} \sim \tau^{2 \alpha-2}, \quad \text { as } \quad t \rightarrow t_{s}-
$$

implying that $\max \left\{\left|\eta_{x}\right|\right\}$ and $\max \left\{u^{2}\right\}$ are proportional to each other as the singularity is approached and their values become unbounded. This is in contrast to the $W_{e} \neq 0$ case, where we found that $\max \left\{\left|\eta_{x x}\right|\right\}$ and $\max \left\{u^{2}\right\}$ being proportional near the singular time. Numerical results confirming (5.21) for the case $\rho=3$ and $\bar{E}_{b}=1$ (recall that $W_{e}=0$ throughout this section) are given in the right panel of Figure 12, and the proportionality of the two quantities as they grow is clearly seen, providing strong support for the self-similar ansatz (5.19).

The scalings (5.19) suggest that as $t \rightarrow t_{s}-$ and touch-up takes place, the minimum layer thickness $\eta_{\min }$ and the corresponding maximum horizontal fluid velocity $u_{\max }$ have the following behavior:

$$
\eta_{\min } \sim\left(t_{s}-t\right)^{3 \alpha-2}, \quad u_{\max } \sim\left(t_{s}-t\right)^{\alpha-1} .
$$

Following the ideas from section 5.1.1, we have used the computations for the case $W_{e}=0, \rho=3, \bar{E}_{b}=1$ in order to find best numerical fits for $\eta_{\min }$ and $u_{\max }$ and hence estimate $t_{s}$ and $\alpha$ (see section 5.1.1 for details). We find that $\eta_{\min }$ approaches zero as $\left(t_{s}-t\right)^{0.39994}$ and $u_{\max }$ blows up as $\left(t_{s}-t\right)^{-0.20312}$, with the singular time being $t_{s} \approx 3.0333$. Scalings $(5.22)$ then imply that $\alpha \approx 0.8$ or $\alpha \approx 0.7969$, respectively, and these approximations of $\alpha$ agree with an error of less than $0.4 \%$. The results of the numerical fitting are included in Figure 13, which shows log-log plots of $\eta_{\min }$ and $u_{\max }$ versus $t_{s}-t$, along with superimposed linear fits utilized to extract $\alpha$.

An estimation of $\alpha$ without the need to know $t_{s}$ numerically, analogous with that of section 5.1.2, where $W_{e} \neq 0$, has also been considered. Introducing the functions $M_{1}=\ln \left(|u|_{\max }\right)=(\alpha-1) \ln \left(t_{s}-t\right)+K_{1}(t), M_{2}=\ln \left(\eta_{\min }\right)=(3 \alpha-2) \ln \left(t_{s}-t\right)+K_{2}(t)$, and $M_{3}=\ln \left(\left|u_{x}\right|_{\max }\right)=-\ln \left(t_{s}-t\right)+K_{3}(t)$, we have for $t$ close to $t_{s}$,

$$
M_{1} \sim(\alpha-1) \ln \left(t_{s}-t\right), \quad M_{2} \sim(3 \alpha-2) \ln \left(t_{s}-t\right), \quad M_{3} \sim-\ln \left(t_{s}-t\right) .
$$

As the computation evolves, the functions $\psi_{13}=1-\frac{M_{1}}{M_{3}}, \psi_{23}=\frac{1}{3}\left(2-\frac{M_{2}}{M_{3}}\right)$, and $\psi_{12}=$ 


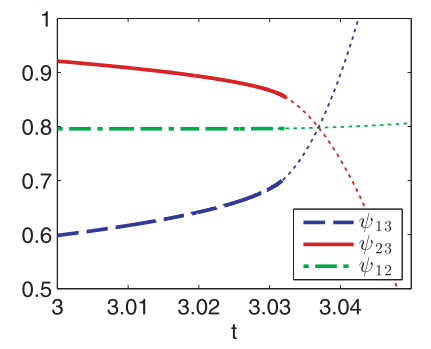

FIG. 14. Evolution of functions $\psi_{13}, \psi_{23}, \psi_{12}$ that tend to $\alpha$ as $t \rightarrow t_{s}$ in the case $W_{e}=0$, $\rho=3, \bar{E}_{b}=1$. We find $\alpha \approx 0.798$.
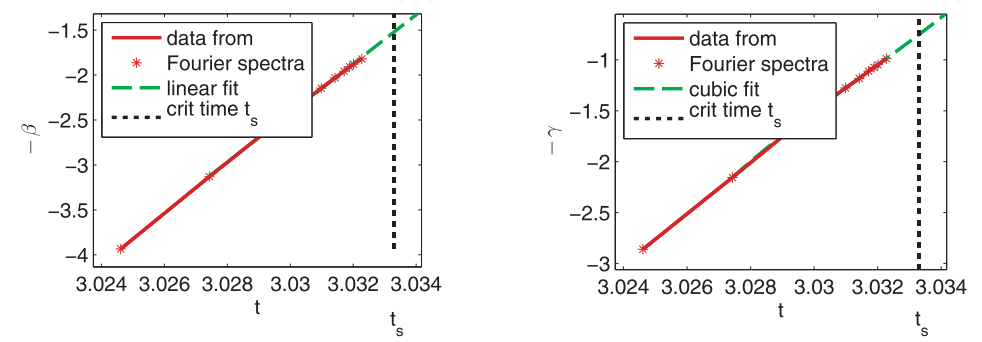

FIG. 15. Extrapolation of the decay rates $\beta$ (left panel) and $\gamma$ (right panel) of the Fourier spectra $\left(-\beta\right.$ and $-\gamma$ are plotted) to $t_{s} \approx 3.0333$ for the case $W_{e}=0, \rho=3, \bar{E}_{b}=1$. We find $\beta=1.5193$ and $\gamma=0.7506$, and, hence, $\alpha \approx 0.80624$ and $\alpha \approx 0.80038$, respectively.

$\left(-1+2 \frac{M_{1}}{M_{2}}\right) /\left(3 \frac{M_{1}}{M_{2}}-1\right)$ should approach $\alpha$ when $t$ gets close to $t_{s}$. The evolution of $\psi_{13}, \psi_{23}, \psi_{12}$ for the case $W_{e}=0, \rho=3, \bar{E}_{b}=1$ studied numerically in this section is shown in Figure 14. Extrapolating the curves using quadratic polynomials gives $\alpha=0.798$ in excellent agreement with the values of $\alpha$ obtained above using a different fitting method. This value of $\alpha$ implies that $\eta_{\min } \approx\left(t_{s}-t\right)^{0.394}$ and $u_{\max } \approx$ $\left(t_{s}-t\right)^{-0.202}$ as $t \rightarrow t_{s}$. To illustrate the robustness of the fitting approach requiring $t_{s}$ (section 5.1.1) with that of the ratios of the functions (5.23) (see section 5.1.2 for details), we have superimposed $\eta_{\min } \approx\left(t_{s}-t\right)^{0.394}$ and $u_{\max } \approx\left(t_{s}-t\right)^{-0.202}$ with the fitting approach results in Figure 13, and agreement is seen to be excellent.

For completeness, we carry out an estimate of $\alpha$ using the spectra of the solutions to infer the spatial structure of the singularity. According to the self-similar solutions, we expect the theoretical results (5.20) to hold. Assuming that the numerical solutions provide the structures $\eta \sim\left|x-x_{s}\right|^{p}$ and $u \sim\left|x-x_{s}\right|^{-q}$ with $p, q>0$, we estimate $p$ and $q$ using the methods described in section 5.1.3, and hence obtain two independently derived values of $\alpha$ given by

$$
\alpha=\frac{2}{3-p}, \quad \alpha=\frac{1}{1+q},
$$

or in terms of the decay rates $\beta(t)$ and $\gamma(t)$ of the Fourier spectra of $\eta$ and $u$ (see (5.15)), we have $\alpha=\frac{2}{4-\beta}, \alpha=\frac{1}{2-\gamma}$ as $t \rightarrow t_{s}$. Extrapolating $\beta(t)$ and $\gamma(t)$ to $t_{s} \approx 3.0333$ as shown in Figure 15 , we obtain $\beta_{s}=1.5193$ and $\gamma_{s}=0.7506$, which imply that $\alpha \approx 0.80624$ or $\alpha \approx 0.80038$, respectively. Both obtained values of $\alpha$ agree well with the value $\alpha=0.798$ found earlier and other previously computed approximations of $\alpha$.

Given the calculated value of $\alpha \approx 0.8$ (and equivalently the values $p \approx 0.5$ and $q \approx 0.25$ from (5.24)), the spatial structure of the singularity in the absence of surface

Copyright @ $\odot$ by SIAM. Unauthorized reproduction of this article is prohibited. 
tension becomes

$$
\eta \sim\left|x-x_{s}\right|^{0.5}, \quad u \sim\left|x_{s}-x\right|^{-0.25}
$$

These terminal states are quite distinct from those with surface tension (see (5.16)); the singularity when a field is present terminates in a cusp with unbounded slopes (i.e., much sharper than when surface tension is present), while the fluid velocity is less singular (the exponent is -0.25 rather than -0.46 when surface tension is present). Once again the long wave models break down in the vicinity of touch-up, and the full Euler equations need to be considered to construct an inner nonslender region.

In the above numerical experiments, we considered the initial condition (4.1) with the stable or unstable wavenumber $k=1$ depending on which values of $W_{e}$, $\rho$, and $\bar{E}_{b}$ were used. We also studied other sets of parameters $W_{e}, \rho$, and $\bar{E}_{b}$ that support several unstable modes, so we could in turn use a $k \neq 1$ mode as an initial condition to follow the dynamics to the singular states. In particular, we took the case with $W_{e}=1, \rho=17$, and $\bar{E}_{b}=0$, for which linear theory predicts the most unstable wavenumber $k_{\max } \approx 2.82$ and a cutoff wavenumber $k_{c}=4$ (i.e., all waves with $k>k_{c}$ are stable). Using the initial condition $\eta(x, 0)=1-\frac{1}{4} \cos (3 x)$ corresponds to a $\frac{2 \pi}{3}$-periodic perturbation. Moreover, this initial condition is close to the most unstable wave. In this case, the interface evolves to touch the wall at six positions while the velocity blows up at these points, as expected. Analyzing the singularity similarly as for the other initial condition, we obtained almost the same value for the exponent $\alpha$. Agreement improves as we get closer to the singularity - in our runs with $\eta_{\min } \approx 0.0123$ the difference between the two values of $\alpha$ was less than $3 \%$. This suggests that the local singular structures described in the paper are not expected to depend on initial conditions.

6. Conclusions. We studied singularity formation in a two fluid system in an infinite channel with an electric field imposed in the horizontal direction and in the plane of the undisturbed fluid interface. The fluids can have different densities and electric permittivities. Gravity and surface tension are taken into account. Of particular interest is the case with the heavier fluid lying initially above the lighter fluid (in the present case, air). The system of evolution equations for the interface and horizontal velocity in the case when the upper layer is thin was derived by the authors in [6], where it was also shown that a strong enough electric field can completely suppress the Rayleigh-Taylor instability. When the electric field is weak or absent, the equations encounter a finite-time singularity at $t=t_{s}$ with the interface touching the upper wall at a point(s) $x=x_{s}$ and the velocity becoming unbounded at $x_{s}$. We study possible similarity solutions of the system when the critical time $t_{s}$ is approached and find that the singularity is of the second type since the order of magnitude analysis cannot determine the exponents uniquely - they depend on a parameter $\alpha$ that needs to be determined numerically. The initial value problem, subject to periodic boundary conditions, is solved for various sets of the physical parameters to obtain numerical solutions at times close to $t_{s}$. Three different approaches are used to estimate $\alpha$ and $t_{s}$. The first two are based on the temporal structure of singularity: one of the methods requires an accurate approximation of $t_{s}$ to estimate $\alpha$, and another does not require a priori knowledge of $t_{s}$ to find $\alpha$. The third approach employs the spatial structure of the singularity and allows us to approximate $\alpha$ by extracting the rates of decay of Fourier coefficients of the solutions at times close to $t_{s}$. All three approaches produce accurate and robust approximations of $\alpha$.

We find that when surface tension is present, $\alpha \approx 0.689$ and $\eta_{\min } \sim\left(t_{s}-t\right)^{4 \alpha-2} \approx$ $\left(t_{s}-t\right)^{0.7560}$ and $u_{\max } \sim\left(t_{s}-t\right)^{\alpha-1} \approx\left(t_{s}-t\right)^{-0.3110}$ for times $t$ near $t_{s}$. The spatial 
structure of the singularity indicates that the leading order singular behavior of the solution near $x_{s}$ is $\eta \sim\left|x-x_{s}\right|^{1.1}, u \sim\left|x-x_{s}\right|^{-0.46}$, i.e., the interface touches the wall with bounded slope but an infinite curvature, while the horizontal velocity blows up. In the presence of surface tension, the electric field term does not enter the leading order asymptotic balances that provide the local self-similar solution. Consequently, in the absence of surface tension the self-similar structures are different and are driven by the electric stresses. In this case, we find $\alpha \approx 0.798$ and $\eta_{\min } \sim$ $\left(t_{s}-t\right)^{3 \alpha-2} \approx\left(t_{s}-t\right)^{0.3940}$ and $u_{\max } \sim\left(t_{s}-t\right)^{\alpha-1} \approx\left(t_{s}-t\right)^{-0.2020}$ near the singular time. The terminal spatial states of the singular solutions now become $\eta \sim\left|x-x_{s}\right|^{0.5}$, $u \sim\left|x-x_{s}\right|^{-0.25}$, which implies that in the absence of surface tension but with the electric field present, the interface develops a cusp at touch-up with unbounded slopes in contrast to the surface tension case. At the same time, the horizontal velocity is less singular as compared to the surface tension case.

In the solutions described here, viscosity has been ignored throughout. It is reasonable to expect viscous effects to enter as the interface gets close to the upper wall, and the solutions found here would provide the appropriate outer problem to be matched onto a viscously dominated region. We note that viscous hanging films that are Rayleigh-Taylor unstable but which also support strong surface tension that regularizes short waves have been studied in detail in [38]. In fact, those authors also include vertical electric fields that enhance the gravitational destabilization. It is found (both analytically and computationally) that the film does not drain to zero thickness in finite time (i.e., the interface does not touch the wall), but instead the thickness decreases to zero algebraically with time, as time tends to infinity. We surmise, therefore, that analogous behavior could be encountered if viscous effects are sufficiently strong. Such analysis is beyond the scope of the present work and is a very challenging multiscale analytical and computational problem that is left for future studies.

\section{REFERENCES}

[1] S. I. Abarzhi, Review of theoretical modelling approaches of Rayleigh-Taylor instabilities and turbulent mixing, Philos. Trans. R. Soc. A, 368 (2010), pp. 1809-1828.

[2] S. I. Abarzhi, S. Gauthier, and K. R. Sreenivasan, Turbulent mixing and beyond: Nonequilibrium processes from atomistic to astrophysical scales, Philos. Trans. R. Soc. A Math. Phys. Eng. Sci., 371 (2013), 20130268.

[3] C. Almarcha, P. Clavin, L. Duchemin, and J. Sanz, Ablative Rayleigh-Taylor instability with strong temperature dependence of the thermal conductivity, J. Fluid Mech., 579 (2007), pp. 481-492.

[4] G. Baker, R. E. Caflisch, and M. Siegel, Singularity formation during Rayleigh-Taylor instability, J. Fluid Mech., 252 (1993), pp. 51-78.

[5] L. L. BARANNYK AND D. T. PAPAGEORGIOU, Fully nonlinear gravity-capillary solitary waves in a two-fluid system of finite depth, J. Engrg. Math., 42 (2002), pp. 321-339.

[6] L. L. Barannyk, D. T. Papageorgiou, and P. G. Petropoulos, Suppression of RayleighTaylor instability using electric fields, Math. Comput. Simulation, 82 (2012), pp. 10081016.

[7] R. BARROS AND W. CHOI, Inhibiting shear instability induced by large amplitude internal solitary saves in two-layer flows with a free surface, Stud. Appl. Math., 122 (2009), pp. 325346 .

[8] R. E. Caflisch And O. F. ORellana, Singular solutions and ill-posedness for the evolution of vortex sheets, SIAM J. Math. Anal., 20 (1989), pp. 293-307.

[9] M. Carrier, C. E. Krook, and L. C. Pearson, Functions of a Complex Variable: Theory and Technique, Hod Books, Ithaca, New York, 1983.

[10] B. Castaing, G. Gunaratne, F. Heslot, L. Kadanoff, A. Libchaber, S. Thomae, X. Z. Wu, S. Zaleski, And G. Zanetti, Scaling of hard thermal turbulence in Rayleigh-Benard convection, J. Fluid Mech., 204 (1989), pp. 1-30.

Copyright @ $\odot$ by SIAM. Unauthorized reproduction of this article is prohibited. 
[11] S. Chandrasekhar, Hydrodynamic and Hydromagnetic Stability, Internat. Ser. Monogr. Phys., Dover, New York, 1981.

[12] W. Choi, A. Goullet, And T.-C. Jo, An iterative method to solve a regularized model for strongly nonlinear long internal waves, J. Comput. Phys., 230 (2011), pp. 2021-2030.

[13] R. Cimpeanu, D. T. Papageorgiou, and P. G. Petropoulos, On the control and suppression of the Rayleigh-Taylor instability using electric fields, Phys. Fluids, 26 (2014), 022105.

[14] N. T. Eldabe, Effect of a tangential electric field on Rayleigh-Taylor instability, J. Phys. Soc. Jpn., 58 (1989), pp. 115-120.

[15] S. Grandison, D. T. Papageorgiou, and J.-M. Vanden-Broeck, Interfacial capillary waves in the presence of electric fields, Eur. J. Mech. B Fluids, 26 (2007), pp. 404-421.

[16] S. Grandison, D. T. Papageorgiou, and J.-M. Vanden-Broeck, The influence of electric fields and surface tension on Kelvin-Helmholtz instability in two-dimensional jets, Z. Angew. Math. Phys., 63 (2012), pp. 125-144.

[17] D. Gueyffier and S. Zaleski, Finger formation during droplet impact on a liquid film, C.R. Acad. Sci. Paris Ser. IIb, 326 (1998), pp. 839-844.

[18] P. Hall and D. T. Papageorgiou, Chaos in a class of solution of the Navier-Stokes equations, J. Fluid Mech., 393 (1999), pp. 59-87.

[19] T. Y. Hou AND R. LI, Computing nearly singular solutions using pseudo-spectral methods, J. Comput. Phys., 226 (2007), pp. 379-397.

[20] M. Hunt, D. T. Papageorgiou, and J.-M. Vanden-Broeck, Electrostatic effects on linear and nonlinear waves in hanging film flows, Procedia IUTAM, 11 (2014), pp. 58-68.

[21] J. D. JACKsON, Classical Electrodynamics, Wiley, New York, 1999.

[22] A. Joshi, M. C. Radhakrishna, and N. Rudraiah, Rayleigh-Taylor instability in dielectric fluids, Phys. Fluids, 22 (2010), 064102.

[23] R. KRAsny, A study of singularity formation in a vortex sheet by the point-vortex approximation, J. Fluid Mech., 167 (1986), pp. 65-93.

[24] D. W. Moore, Spontaneous appearance of a singularity in the shape of an evolving vortex sheet, Proc. R. Soc. Lond. Ser. A Math. Phys. Eng. Sci., 365 (1979), pp. 105-119.

[25] D. T. Papageorgiou, Analytical description of the breakup of liquid jets, J. Fluid Mech., 301 (1995), pp. 109-132.

[26] D. T. Papageorgiou, On the breakup of viscous liquid threads, Phys. Fluids, 7 (1995), pp. 1529 1544.

[27] D. T. Papageorgiou, P. G. Petropoulos, and J.-M. Vanden-Broeck, Gravity capillary waves in fluid layers under normal electric fields, Phys. Rev. E, 72 (2005), 051601.

[28] D. T. Papageorgiou and J.-M. Vanden-Broeck, Large-amplitude capillary waves in electrified fluid sheets, J. Fluid Mech., 508 (2004), pp. 71-88.

[29] M. C. Pugh And M. J. Shelley, Singularity formation in thin jets with surface tension, Comm. Pure Appl. Math., 51 (1998), pp. 733-795.

[30] L. RAYLEIGH, Investigation of the character of the cquilibrium of an incompressible heavy fluid of variable density, Proc. Lond. Math. Soc., S1-14 (1883), pp. 170-177.

[31] J. Sanz, J. Ramirez, R. Ramis, R. Betti, and R. P. J. Town, Nonlinear theory of the ablative Rayleigh-Taylor instability, Phys. Rev. Lett., 89 (2002), pp. B367-B380.

[32] D. H. Sharp, An overview of Rayleigh-Taylor instability, Phys. D, 12 (1984), pp. 3-18.

[33] M. Siegel, A study of singularity formation in the Kelvin-Helmholtz instability with surface tension, SIAM J. Appl. Math., 55 (1995), pp. 865-891.

[34] C. Sulem, P. L. Sulem, And H. Frisch, Tracing complex singularities with spectral methods, J. Comput. Phys., 50 (1983), pp. 138-161.

[35] G. TAYLOR, The instability of liquid surfaces when accelerated in a direction perpendicular to their planes. I, Proc. R. Soc. Lond. Ser. A., 201 (1950), pp. 192-196.

[36] B. S. Tilley, P. G. Petropoulos, and D. T. Papageorgiou, Dynamics and rupture of planar electrified liquid sheets, Phys. Fluids, 13 (2001), pp. 3547-3563.

[37] G. Tryggvason, Numerical simulations of the Rayleigh-Taylor instability, J. Comput. Phys., 75 (1988), pp. 253-282.

[38] D. Tseluiko and D. T. Papageorgiou, Nonlinear dynamics of electrified thin liquid films, SIAM J. Appl. Math., 67 (2007), pp. 1310-1329.

[39] C. P. Verdon, R. L. McCrory, R. L. Morse, G. R. Baker, D. I. Meiron, and S. A. Orszag, Non-linear effects of multifrequency hydrodynamic instabilities on ablatively accelerated thin shells, Phys. Fluids, 25 (1982), pp. 1653-1674.

[40] Q. Wang, S. Mählmann, AND D. T. PAPAGeorgiou, Dynamics of liquid jets and threads under the action of radial electric fields: Microthread formation and touchdown singularities, Phys. Fluids, 21 (2009), 032109.

Copyright (c) by SIAM. Unauthorized reproduction of this article is prohibited. 